\title{
ORDINARY \\ DIFFERENTIAL \\ EQUATIONS WITH \\ APPLICATIONS
}




\section{SERIES ON APPLIED MATHEMATICS}

Editor-in-Chief: Frank Hwang

Associate Editors-in-Chief: Zhong-ci Shi and U Rothblum

Vol. 1 International Conference on Scientific Computation

eds. T. Chan and Z.-C. Shi

Vol. 2 Network Optimization Problems - Algorithms, Applications and Complexity

eds. D.-Z. Du and P. M. Pandalos

Vol. 3 Combinatorial Group Testing and Its Applications by D.-Z. Du and F. K. Hwang

Vol. 4 Computation of Differential Equations and Dynamical Systems eds. K. Feng and Z.-C. Shi

Vol. 5 Numerical Mathematics eds. Z.-C. Shi and T. Ushijima

Vol. 6 Machine Proofs in Geometry by S.-C. Chou, X.-S. Gao and J.-Z. Zhang

Vol. 7 The Splitting Extrapolation Method by C. B. Liem, T. Lü and T. M. Shih

Vol. 8 Quaternary Codes by Z.-X. Wan

Vol. 9 Finite Element Methods for Integrodifferential Equations by C. M. Chen and T. M. Shih

Vol. 10 Statistical Quality Control - A Loss Minimization Approach by $D$. Trietsch

Vol. 11 The Mathematical Theory of Nonblocking Switching Networks by F. K. Hwang

Vol. 12 Combinatorial Group Testing and Its Applications (2nd Edition) by D.-Z. Du and F. K. Hwang

Vol. 13 Inverse Problems for Electrical Networks by E. B. Curtis and J. A. Morrow

Vol. 14 Combinatorial and Global Optimization eds. P. M. Pardalos, A. Migdalas and R. E. Burkard

Vol. 15 The Mathematical Theory of Nonblocking Switching Networks (2nd Edition) by F. K. Hwang

Vol. 16 Ordinary Differential Equations with Applications by S. B. Hsu

Vol. 17 Block Designs: Analysis, Combinatorics and Applications by D. Raghavarao and L. V. Padgett 


\section{Published by}

World Scientific Publishing Co. Pte. Ltd.

5 Toh Tuck Link, Singapore 596224

USA office: 27 Warren Street, Suite 401-402, Hackensack, NJ 07601

UK office: 57 Shelton Street, Covent Garden, London WC2H 9HE

\section{Library of Congress Cataloging-in-Publication Data \\ Hsu, Sze-Bi, 1948- \\ Ordinary differential equations with applications / Sze-Bi Hsu. \\ p. cm. -- (Series on applied mathematics ; v. 16) \\ Includes bibliographical references and index. \\ ISBN 981-256-319-9 (alk. paper) \\ 1. Differential equations. I. Title. II. Series.}

QA372.H85 2005

$515 \cdot .352--d c 22$

2005044280

\section{British Library Cataloguing-in-Publication Data}

A catalogue record for this book is available from the British Library.

\section{Copyright $\odot 2006$ by World Scientific Publishing Co. Pte. Ltd.}

All rights reserved. This book, or parts thereof, may not be reproduced in any form or by any means, electronic or mechanical, including photocopying, recording or any information storage and retrieval system now known or to be invented, without written permission from the Publisher.

For photocopying of material in this volume, please pay a copying fee through the Copyright Clearance Center, Inc., 222 Rosewood Drive, Danvers, MA 01923, USA. In this case permission to photocopy is not required from the publisher. 


\section{Contents}

1 INTRODUCTION 1

1.1 Where do ODEs arise . . . . . . . . . . . . . 1

2 FUNDAMENTAL THEORY 9

2.1 Introduction and Preliminaries . . . . . . . . . . . 9

2.2 Local Existence and Uniqueness of Solutions of I.V.P. . . . 12

2.3 Continuation of Solutions . . . . . . . . . . . . 21

2.4 Continuous Dependence Properties . . . . . . . . . . . 24

2.5 Differentiability of Initial Conditions and Parameters . . . . 27

2.6 Differential Inequalities $\ldots \ldots \ldots \ldots \ldots \ldots$

2.7 Exercises . . . . . . . . . . . . . . . 35

3 LINEAR SYSTEMS

3.1 Introduction . . . . . . . . . . . . . . 41

3.2 Fundamental Matrices . . . . . . . . . . . . . . . 42

3.3 Linear Systems with Constant Coefficients . . . . . . . . . 46

3.4 Two-Dimensional Linear Autonomous Systems . . . . . . 55

3.5 Linear Systems with Periodic Coefficients . . . . . . . . 59

3.6 Adjoint Systems . . . . . . . . . . . . . . 67

3.7 Exercises . . . . . . . . . . . . . . 71

4 STABILITY OF NONLINEAR SYSTEMS

4.1 Definitions . . . . . . . . . . . . . . . . . 77

4.2 Linearization . . . . . . . . . . . . . . . . 79

4.3 Saddle Point Property: Stable and Unstable Manifolds . . . 88

4.4 Orbital Stability . . . . . . . . . . . . . . . . . 99

4.5 Travelling Wave Solutions . . . . . . . . . . . . . 106

4.6 Exercises . . . . . . . . . . . . . . . . . . . 114 
5 METHOD OF LYAPUNOV FUNCTIONS 117

5.1 An Introduction to Dynamical Systems . . . . . . . . 117

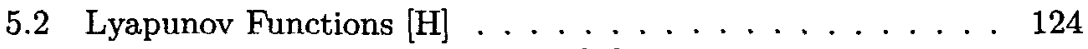

5.3 Simple Oscillatory Phenomena: $[\mathrm{H}] \ldots \ldots \ldots \ldots$

5.4 Exercises . . . . . . . . . . . . . . . 138

6 TWO-DIMENSIONAL SYSTEMS 143

6.1 Poincaré-Bendixson Theorem . . . . . . . . . . . . 143

6.2 Levinson-Smith Theorem . . . . . . . . . . . . . . 154

6.3 Hopf Bifurcation . . . . . . . . . . . . . . 163

6.4 Exercises . . . . . . . . . . . . . . 168

7 SECOND ORDER LINEAR EQUATIONS 171

7.1 Sturm's Comparison and Sturm-Liouville Boundary Value Problems ................... 171

7.2 Distributions . . . . . . . . . . . . . . . . . . . . 179

7.3 Green's Function . . . . . . . . . . . . . . . . . . 181

7.4 Fredholm Alternative for 2nd Order Linear Equations . . . 187

7.5 Exercises . . . . . . . . . . . . . . . . . 189

8 THE INDEX THEORY AND BROUWER DEGREE 193

8.1 Index Theory in the Plane . . . . . . . . . . . . 193

8.2 Introduction to the Brouwer Degree in $R^{n} \ldots \ldots \ldots 202$

8.3 Exercises . . . . . . . . . . . . . . 206

9 INTRODUCTION TO PERTURBATION METHODS 209

9.1 Regular Perturbation Methods . . . . . . . . . . 209

9.2 Singular Perturbations : Boundary Value Problems . . . . 216

9.3 Singular Perturbation : Initial Value Problems . . . . . . 221

9.4 Exercises . . . . . . . . . . . . . . . . 234

$\begin{array}{ll}\text { BIBLIOGRAPHY } & 237\end{array}$

INDEX $\quad 241$ 


\section{Preface}

Ordinary Differential Equations (ODE) is a well-developed discipline. It is a classical subject for most graduate students to study if they want to learn analysis and applied mathematics. There are many standard texts of ODE, for instance, the books, Coddington and Levinson [CL], Hartman [Ha], J. Hale [H], Miller and Michel [MM]. Based on my past twenty years of teaching experience of graduate course in ODE, I find that for most graduate students these books are either too difficult to understand or too abstract to follow. This is the reason why I have decided to write my own ODE lecture notes. This book is aimed to present the classical ODE materials in an easy way with rigorous proofs. In this text many examples from mathematical biology and physical science to interpret the meanings and the applications of the theorems have been given. Besides the classical materials, Brouwer degree in finite dimensional space following the index theory has been discussed in Chapter 8. Brouwer degree is a powerful topological tool for studying the nonlinear analysis. Introducing Brouwer degree paves the way to the Leray-Schauder degree in infinite dimensional space. In Chapter 9 we introduce perturbation method, the regular perturbation and singular perturbation methods which are very important in applied mathematics.

This book is designed for a one-year graduate course. Let's briefly state the contents of each chapter in this book.

Chapter 1: Introduction. In this chapter important examples of nonlinear systems of ODEs from physical science and mathematical biology have been introduced.

Chapter 2: Fundamental Theory. In this chapter I first prove the local existence and uniqueness of solutions of initial value problem of ODE. Then the continuation of the solutions and the global existence of the solutions are studied. The continuous dependence on initial conditions and parameters is proved. Applying continuous dependence on initial conditions and parameters gives the results of scalar differential inequalities. The differen- 
tial inequalities for systems are also discussed.

Chapter 3: Linear Systems. In this chapter we discuss the fundamental matrices of general linear systems $x^{\prime}=A(t) x$. Then for the linear system with constant coefficients $x^{\prime}=A x$ we introduce exponential matrix $e^{A t}$ as a fundamental matrix. For periodic linear system $x^{\prime}=A(t) x$, $A(t+\omega)=A(t), \omega>0$, we study the structure of the solutions by proving Floque's Theorem. For two-dimensional linear autonomous $\left(\begin{array}{l}x \\ y\end{array}\right)^{\prime}=$ $\left(\begin{array}{ll}a & b \\ c & d\end{array}\right)\left(\begin{array}{l}x \\ y\end{array}\right)$, we introduce the important notions of node, focus, center and saddle. Similar to linear algebra, we introduce adjoint systems of $x^{\prime}=A x$. We obtain important results similar to the fundamental theorem of linear algebra.

Chapter 4: Stability of Nonlinear Systems. In this chapter we introduce concepts of stability, asymptotic stability and instability of an equilibrium solution. To verify these stabilities, we introduce the method of linearization by checking the eigenvalues of variational matrix. When the equilibrium is a saddle, we prove the existence of stable and unstable manifolds. For periodic orbit, we introduce the concept of orbit stability which is different from the asymptotic stability. The results related to Floquet multipliers have been discussed in Chapter 3 . In section 4.5 we study the existence of travelling wave solutions of the well-known partial differential equations, the Fisher's equation and the bistable equation by applying the Stable Manifold Theorem.

Chapter 5: Method of Lyapunov Functions. In this chapter we first introduce the concept of dynamical system, $\alpha$-limit set, $\omega$-limit set and their properties in abstract setting. In fact, autonomous ODE system is an important example of dynamical system. Then we discuss Lyapunov functions and their use in determining the global asymptotic stability or the domain of attraction of a locally stable equilibrium. We prove the LaSalle's invariance principle which explains how to apply Lyapunov function to locate $\omega$-limit set.

Chapter 6: Two-Dimensional Systems. In this chapter we prove one of the most important theorems in nonlinear dynamics, Poincaré-Bendixson Theorem which states that a bounded trajectory of a two-dimensional system either converges to an equilibrium or approaches a limit cycle. We also discuss Dulac Criterion which provides a method to eliminate the existence of periodic solutions. In section 6.2 we discuss the Levinson-Smith Theorem which proves the uniqueness of limit cycle for Lienard equation. An important example of Lienard equation is van der Pol equation. We discuss the relaxation property of the van der Pol oscillators. In section 6.3, we introduce Hopf Bifurcation Theorem which is an important tool to detect 
the existence of periodic solution for autonomous system $x^{\prime}=f(\lambda, x)$ where $\lambda$ is a bifurcation parameter.

Chapter 7: Second Order Linear Equations. In this chapter we first discuss the Sturm's Comparison Theorem which is an important tool to study oscillation properties of a solution of a second order linear equation. Similar to the eigenvalue problems $A x=\lambda x$, we consider the Sturm-Liouville boundary value problem and prove the existence of infinitely many discrete eigenvalues and show that the corresponding eigenfunctions satisfy the "node" properties. For second order nonlinear equation $x^{\prime \prime}=f\left(t, x, x^{\prime}\right)$, $x(a)=x_{0}, x(b)=x_{1}$, we introduce Green's function so that we convert the boundary value problem into an integral equation.

Chapter 8: The Index Theory and Brouwer Degree. In this chapter we first introduce the Index $I_{f}(C)$ for a vector field $f$ in the plane and a simple closed curve $C$. Then we prove various properties of index and apply index theory to prove fundamental theorem of algebra and Brouwer fixed point theorem. In section 8.2 we generalize the index $I_{f}(C)$ to the topological degree in finite dimensional space, the Brouwer degree in $R^{n}$. Topological degree is an important tool in studying nonlinear problems.

Chapter 9: Introduction to Regular and Singular Perturbation Methods. Perturbation methods are very important in applied mathematics. In this chapter we explain why regular perturbation method works by using Implicit Function Theorem and Fredholm Alternatives. Then we discuss singular perturbation methods for both boundary value problems and initial value problems.

Acknowledgments: I would like to express my gratitude to my wife Taily for her support in the past ten years; to my former Ph.D. thesis adviser Professor Paul Waltman for his encouragement to write this book. I want to thank my colleagues Professor Wen-Wei Lin, Professor Shin-Hua Wang, Professor Shuh-Jye Chern of National Tsing-Hua University, Professor Chin-An Wang, Professor Dong-Ho Tsai of National Chung-Cheng University for using this text in their graduate ODE courses and for giving me some suggestions to improve the text. I also want to thank my post doctor Dr. Cheng-Che Li and my Ph.D student Yun-Huei Tzeng for their patience to proofreading the draft. Finally I especially want to thank Miss Alice Feng for typing this text in the past three years. Without her help, it is impossible to have this book published.

Sze-Bi Hsu

National Tsing-Hua University

Hsinchu, Taiwan

Aug. 30, 2005 